\title{
Experimental Study of the Stress Level at the workplace using an Smart Testbed of Wireless Sensor Networks and Ambient Intelligence techniques
}

\author{
F. Silva +, T. Olivares *, F. Royo *, M.A. Vergara *, and C. Analide + \\ + University of Minho, Braga, Portugal \\ * Albacete Research Institute of Informatics \\ University of Castilla-La Mancha, 02071 Albacete, Spain \\ fabiosilva, analide@di.uminho.pt, teresa,froyo@dsi.uclm.es,
}

\begin{abstract}
This paper combines techniques of ambient intelligence and wireless sensor networks with the objective of obtain important conclusions to increase the quality of life of people. In particular we oriented our study to the stress at the workplace, because stress is a leading cause of illness and disease. This article presents a wireless sensor networks obtaining information of the environment, a pulse sensor obtaining hear rate values and a complete data analysis applying techniques of ambient intelligence to predict stress from these environment variables and people attributes.Results show promise on the identification of stressful situations as well as stress inference through the use of predictive algorithms.
\end{abstract}

Keywords: Ambient Intelligence, Intelligent Environments, Wireless Sensor networks, Body Area Networks, Environmental Monitoring, Stress Detection

\section{Introduction}

Ambient Intelligence $(\mathrm{AmI})$ is considered one of three emergent technologies: Ubiquitous Computing, Ubiquitous Communication and Intelligent User Interfaces. AmI systems aim to change how people interact with technology and the environment integrating concepts from psychology, social sciences and artificial intelligence to increase the quality of life. AmI makes this possible by anticipating and predicting future needs and desires while taking in consideration aspects like safety, economy and comfort. One concept usually linked to AmI is ubiquitous computing, a concept proposed in [1] by Mark Weiser. In this environment, computational units are embedded in its surroundings functioning and hidden from view.

Directly connected with ambient intelligence is also how the sensing of the environment is done. In order to deliberate which actions an intelligent system 
may do in an environment it is necessary to obtain the information of the environment needs. Significant levels of data must be constantly and ubiquitously collected to provide the data and information needed.

Body Area Networks (BANs) allow the integration of miniaturized and low power sensor nodes in, on, or around the human body to monitor body functions and the surrounding environment [2][3]. BANs provide long term health monitoring of patients under natural physiological states without constraining their normal activities. The typical architecture of a BAN includes sensor nodes deployed in, on or around the body and a BAN coordinator that gathers data coming from all the sensor nodes. This BAN coordinator can store the information for further analysis or can forward it to an intermediate network that serves as a gateway to send the data through the Internet to the expert user (i.e., nurses, doctors, ...) placed in a smart space. Our experiments will use a wireless sensor testbed to emulate this intermediate network. A testbed of wireless sensor nodes provides a reliable platform where to test new protocols and applications in a controlled way.

BANs enable continuous measurement of physiological parameters, such as heart rate, muscular tension, skin conductivity, breathing rate and volume, during the daily life of a user. Those parameters can be combined with contextual information extracted from the environment through wireless sensor networks (temperature, humidity, light, etc.), and all together could be used to infer emotions, mood, depression, and levels of stress and anxiety. Using BANs for emotion, mood, stress or depression recognition is an open research problem and requires development of novel signal processing techniques to interpret and fuse the data collected by multiple sensors [4].

In this work we are going to use a testbed of wireless sensor networks and a BAN. The testbed of 43 fixed nodes is continuously gathering different environment parameters (temperature, humidity, light and CO2). The BAN, composed of a pulse sensor node, is continuously gathering heart rate values. This two networks work together with the objective of detecting possible situations of stress at the workplace using ambient intelligence techniques. This work join two interesting research lines (sensor networks and ambient intelligence) with the purpose of achieve innovative conclusions.

According the World Health Organization a healthy job is likely to be one where the pressures on employees are appropriate in relation to their abilities and resources, to the amount of control they have over their work, and to the support they receive from people who matter to them. As health is not merely the absence of disease or infirmity but a positive state of complete physical, mental and social well-being, a healthy working environment is one in which there is not only an absence of harmful conditions but an abundance of health-promoting ones [5].

Environment and physical working conditions are important organizational risk factors [6]. Our research takes the evaluation of different important parameters related with environment and physical working conditions, using a testbed of wireless sensor network and the extension of the testbed, with sensors installed 
in mobile elements (BANs). The whole wireless network transmits the data to the right base station to store data for a following data analysis. This paper focuses on both, the evaluation with the tesbed and the data analysis.

The rest of this paper is organized as follows: Section 2 summarizes some related work. The testbed used is detailed in Section 3. Section 4 describes the analysis of environment and user data. Finally, Section 5 gives some concluding remarks and interesting future works.

\section{Previous work}

\subsection{Stress monitoring using wireless sensor networks}

There are some interesting works about emotion recognition and stress monitoring with wireless sensors. In [7] we can see a wearable system for ambulatory stress monitoring recording a number of physiological variables known to be influenced by stress. This system is not ZigBee based, it employs a radio module from TI (Texas instruments Inc.), a connection-based low-power lightweight sensor network protocol for small RF networks supporting two basic topologies: strictly peer-to-peer and a star topology. The authors use a star topology where all sensor (leaf nodes) are connected to a sensor hub (the root). If this root node fails the system would support permanent disconnections. Regarding this issue, our mobile nodes send data to the testbed nodes actuating as parents. If a failure is produced in one node, any other node can act like a parent node. In [8] author gathers values from a skin temperature sensor, a heart rate sensor and a skin conductance sensor. The signals from the sensors are input into a microcontroller where all the processing takes place and carried out though ZigBee technology. Data are stored in a computer it is stored for data analysis and feature extraction for emotion recognition. The four basic emotions observed in this project are happy, sad, angry and neutral. The system can be used by anyone sitting within a range of $30 \mathrm{~m}$ of a computer. The sensor used on this system are not too much "wearable". There is an special surface with sensors where a person has to put his hand. Subjects chosen to take part in the experiments have are sitting for neutral state.

\subsection{Ambient intelligence techniques}

Monitor people well being and increases in stress are with the problems covered by ambient intelligence and ubiquitous monitoring. As such, there are model proposals to explain and potentially predict any undesirable effects considering a series of factors believed to influence user/occupant behaviour [9]. Other approaches, relate environment attributes to people attributes such as heart rate which may be used to infer thermal comfort levels and detect stress induced by environment attributes and unoptimised values [10].

In Paola et al. [11], ambient intelligence is used within a sensor network testbed to derive useful from sensor spread across the environment. The data 
retrieved from sensors is used to derive rich information such as user attendance, perceptible temperature, user activity and user preferences. All this information is learned unobtrusively by the system fusing sensor data without requiring user attention or direct user input.

Learning in ambient intelligence is often made using machine learning algorithms such as time series algorithms, evolutionary algorithms and statistical inference. These methods sanction the acquiring of past and current trends and predict future results. From information assembled from different environments, machine learning techniques may derive models of behaviour and interaction based on specialized backgrounds (e.g., users, environment, social interaction or consumption). For such analysis the use of statistical programs such as $\mathrm{R}$ [12] and machine learning workbenches such as Weka [13] is common. Such knowledge about environment and user attributes is usually stored in knowledge databases which are used in deliberative actions or to derive reactive rules to be applied in the system.

\section{The testbed as a powerful and continuous smart tool}

The Albacete Research Institute of Informatics (I3A) has its own testbed of wireless sensor network deployed in its facilities, known as I3ASensorBed [14]. This allows researchers to test in controlled environments. The testbed consists of 43 fixed nodes including temperature, humidity and light sensors, opened/closed doors and windows, movements, air condition (CO, $\mathrm{CO} 2$ and dust) and power consumption. All these sensors allow I3ASensorBed monitor weather conditions and the possibility to add mobile nodes with additional sensor, as hearth [15] rate, the status of workers in a workplace.The distributed nodes are TelosB from Crossbow, TmoteSky from Sentilla and MTM-CM5000-MSP from Maxfor. It also uses nT-A3500 computers from Foxconn, known as supernodes, to redirect data. To facilitate re-program of nodes and debugging of tests, nodes are connecting through USB cable to the nearest supernode, this supernode is the responsible to redirect information the central server. Along with the existing wiring, the nodes have the standard 802.15.4 (ZigBee) for wireless communications emulating realistic environments. The use of the testbed that covers a large work area with multiple workers allows the collection and study of data that is of interest in several areas, especially in the creation of an appropriate working environment for the workers. This is an ideal smart space to contribute to the measurement and experimentation of people health, safety and well-being

\section{Analysis of environment and user data}

In this section the use of ambient intelligence to unobtrusively detect and assess stress situations is made using information gathered by the sensor network detailed in section 3 . The data was collected under real work conditions from an wireless pulse sensor, and embedded temperature, luminosity and humidity sensor for periods of time ranging from 10 minutes to 2 hours. Using these values, 
an heart rate change algorithm was validated and used to learn about stressful conditions in the environment.

\subsection{Preliminary Study}

It is possible to observe that values for environment attributes values are constantly changing throughout the day, see figure 1(a), and it even possible to detect human actions to correct values such as luminosity during some periods of the day, see figure 1 (b). Those corrections are made with artificial lightning to increase luminosity values during work hours and reach comfortable values for people. Such action are taken in order to decrease stress caused by poor environments and facilitate the activities being made.

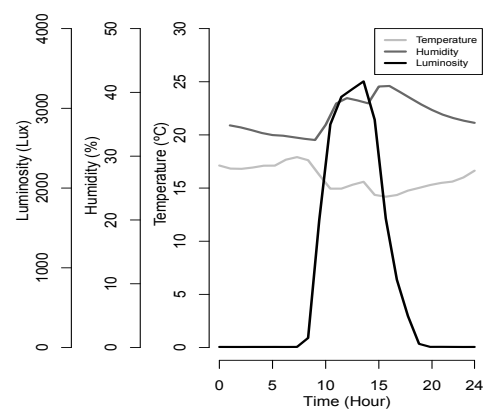

(a) Temperature, Luminosity \& Humidity

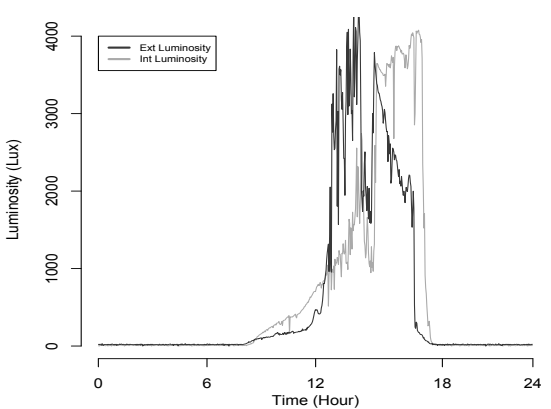

(b) Artificial \& Natural Luminosity

Fig. 1: Environment Monitoring

In the literature review, it is possible to infer a range of ideal values for attributes that while not being directly responsible for health risks, they should increase stress on people. Table 1 demonstrates the use of information about the activity are performing and the values for luminosity deemed acceptable.

Table 1: Luminosity values required by activity

\begin{tabular}{lc}
\hline Activity & Luminosity Required \\
\hline \hline Short Visit & {$[50,100]$} \\
Working areas with few visual tasks & {$[100,150]$} \\
Office Work & {$[250,500]$} \\
Mechanical Workshops, Office Landscapes & 750 \\
Normal Detailed Work & 1000 \\
Detailed Work & {$[1500,2000]$} \\
\hline
\end{tabular}

Likewise, thermal comfort is an area of study that has seen important contributions to assess thermal discomfort and thermal stress in people. The equation of human heat balance is one theory commonly used to derive algorithms that capture the thermal sensation from people, rather than just environment temperature. One theory based on these principles is the Predictive Mean Vote (PMV), a scale from -3 to 3 that classifies thermal sensation and has a correspondence 
to thermal discomfort [16]. Other approach uses an index that translates temperature readings in an environment to a set of stable conditions inside a room environment obtaining what is known as Physiological Equivalent Temperature (PET) [17]. Relationships from both algorithm are explored in Table 2, as well as, indication of thermal stress. The relationship with real temperature values in the environment is dependent on variables such as air speed, clothing, temperature and humidity which change human thermal perception. Studies confirming an influence of thermal conditions and variations in heart rate in people are found in Liu et al, where a medical exam ECG to confirm such relationship between these attributes [10].

Table 2: Temperature Perception

\begin{tabular}{lccc}
\hline PMV & PET & Thermal Sensation & Stress Sensation \\
\hline \hline$<-3.5$ & $<4$ & Very Cold & Extremely cold stress \\
{$[-3.5,-2.5]$} & {$[4,8]$} & Cold & Strong cold stress \\
{$[-2.5,-1.5]$} & {$[8,12]$} & Cool & Moderate cold stress \\
{$[-1.5,-0.5]$} & {$[12,16]$} & Slightly cool & Slight cool stress \\
{$[-0.5,0.5]$} & {$[16,24]$} & Neutral & No thermal stress \\
{$[0.5,1.5]$} & {$[24,28]$} & Slightly warm & Slight warm stress \\
{$[1.5,2.5]$} & {$[28,32]$} & Warm & Moderate warm stress \\
{$[2.5,3.5]$} & {$[32,36]$} & Very Hot & Strong warm stress \\
$>3.5$ & $>36$ & Very Hot & Extremely warm stress \\
\hline
\end{tabular}

In order to validate stress levels with thermal and luminosity values, values from heart rate of people can be used to evaluate if significant changes are occurring.

The use of different sensors enables the application of sensor fusion projects empowered by sensor networks that produce information contextualized to the environment and the people on it. As such, a number of preliminary tests conducted in order to assess the viability of the analysis being undertaken. For these analysis, a test subject was monitored in different thermal situations, in comfortable situation and felling cold. The statistical t-tests were conducted assuming a normal distribution for the values of the heart rate, a 95\% significance value and hypothesis concerning an increase, decrease and no change in the average heart rate of the test subject. These tests found statistical evidence that heart rate increased when on the situation where the test subject felt cold with a $p<0.05$, thus accepting the hypothesis that the heart rate average increased. It is important to notice that in these tests, the test subject did not change its emotional state or any other factor that could affect heart rate values and as such these changes in heart rate averages are concluded to have been caused from environment induced stress that raises the test subject heart rate. Such analysis corroborates other studies that point the same developments in similar conditions and validates the ability for the testbed to produce significant values that can be used in the analysis of stress using values from existing sensors [10]. The same approach with similar sensors was also developed to infer people emotional states from skin temperature sensors and heart rate sensors [18], adding evidence that people react to changes in values of attributes such as temperature. 


\subsection{Detection of Change in Attribute Values}

In order to detect changes in monitored attributes it is necessary to identify points in time that show increases or decreases that have statistical significance for the analysis. Taking heart rate of people as an example, the statistical t-test assuming a normal distribution for heart rate values can be use to create a time series algorithm to detect changes in the average of heart rate. The approach undertaken uses windows of data gathered by a testbed and continuously assesses whether or not the average heart rate is increasing, decreasing or neutral over windows of data with t-test with a significance of $95 \%$, see figure 2 .

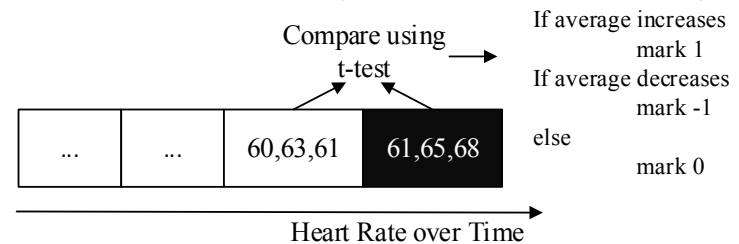

Fig. 2: Window Analysis

Using this strategy it is possible to create a counter variable, adding the all the marks created over time, that provides a contextual information on the balance between increases and decreases in heart rate. An illustration of these counters can be observed in figure 3(a). Substituting the counter value for the average heart rate being used we can produce the same graph but in relationship to real heart rate values, figure 3(b).

The size of the windows of data is important as it influences the number of detections. Big windows detect less changes while smaller windows detect more changes. Moreover, it is also observed that the bigger the window of data the more stable change detections are. Table 3 demonstrates the number of detections using different windows of data for different attributes monitored in the environment.

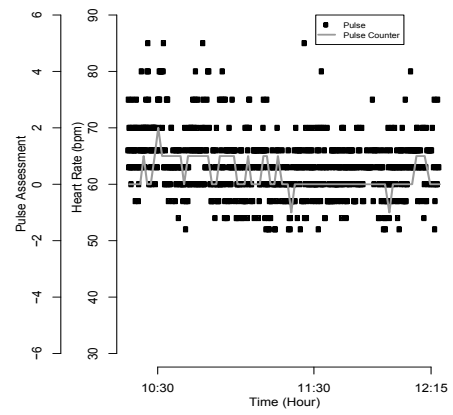

(a) Heart Rate vs Change Counter

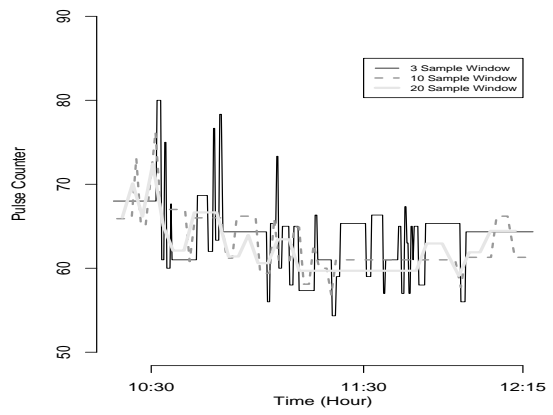

(b) Heart Rate Average Detection over Different Windows of Data

Fig. 3: Heart Rate Monitoring and Assessment 
Table 3: Number of change detection by windows size and attribute

\begin{tabular}{ccccc}
\hline Size of Window Heart Rate & \multicolumn{4}{c}{ Temperature Luminosity Humidity } \\
\hline \hline 3 & 41 & 306 & 104 & 281 \\
10 & 22 & 79 & 49 & 77 \\
20 & 15 & 36 & 24 & 36 \\
\hline
\end{tabular}

\subsection{Predicting Stress through Environment and People Attributes}

A person's heart rate is influenced by a number of factors such as emotional state, stress or physical activity. In the study presented, evidence suggests that environmental variables affect heart rate. As it was presented, comfort values for attributes such as temperature, luminosity or humidity affect the heart rate of people inside an environment. Furthermore, it is possible to link changes in temperature, luminosity and humidity with changes in people's heart rate thus validating the use of comfort values for human well being and stress reduction.

Using datasets created using the attributes monitored from the environment and change markers derived from the attribute change detection algorithm it is possible to train predictive classifiers to predict according to current environment condition how people heart rate will respond. For instance using classifier algorithm from the Weka workbench [13] it is possible to assess with relative precision whether the average heart rate will increase, decrease or maintain. Aside the use of a counter variable to the balance between changes, indicator marking the moment an increase and decrease on the average of each attribute, temperature, humidity luminosity and heart rate as well as the 1 heart rate value. With the dataset generated, some experiments were used on machine learning algorithms, namely the Naive Bayes and J48 algorithms to predict the increases and decreases in heart rate average using. The first algorithm is probabilistic and it builds conditional probability structure to classify instances of data. On the other hand, the $\mathrm{J} 48$ is an implementation of the C4.5 decision tree algorithm which uses an decision tree to classify instances. Both algorithm provide an internal structure that is understandable an may be used to see how changes in attribute values change the output attribute.

Table 4: Heart rate change prediction from environment attributes Data window Algorithm Correct Correct Up Correct No Change Correct Down

\begin{tabular}{lccccc}
\hline & & & & \\
3 & Naives Bayes & $88.7 \%$ & $0 \%$ & $100 \%$ & $0 \%$ \\
3 & J48 & $88.5 \%$ & $0 \%$ & $99.7 \%$ & $0 \%$ \\
10 & Naives Bayes & $63.25 \%$ & $0 \%$ & $77.5 \%$ & $36.3 \%$ \\
10 & J48 & $72.5 \%$ & $0 \%$ & $100 \%$ & $0 \%$ \\
20 & Naives Bayes & $62.2 \%$ & 37.5 & $77.2 \%$ & $37.5 \%$ \\
20 & J48 & $70.3 \%$ & $57.1 \%$ & $86.3 \%$ & $37.5 \%$ \\
\hline
\end{tabular}

Table 4 demonstrates the results from a cross-validation test on the trained classifiers. It is possible to observe that dataset with lower values for the window 
of data yield poor predictive values, however the larger the windows the better the predictive ability. These results indicate that a there may exist a balance between the number of detected changes and the predictability of the heart rate changes. Smaller windows identify more changes but are not easy to predict, larger windows give fewer change detentions but improve predictive tasks.

\subsection{Analysis}

Analysing the results presented, it is possible to correlate heart rate of people with environment conditions, as non-comfortable conditions result in higher heart rate suggesting discomfort to the user. The window analysis used with tests with statistical significance has shown to identify changes in the heart rate condition of people. However, the results also evidence that there are some balances that must taken into consideration when building systems for monitoring and identification of stress induced by the environment. Smaller windows of data result in less stable analysis with more change identifications and less predictive accuracy in the algorithms tests. On the other hand, bigger windows of data decrease the number of change identifications and increase the predictive accuracy of the system. This balance between the size of the window of data, number of change identifications and predictive accuracy determines the overall sensitivity and predictive accuracy of the system. Computational analysis show that it is possible for the sensor node inside the sensor network to handle the workload from detecting change in attribute averages and performing predictions using trained classifiers. However, the initial classifier training should be handled by a central server with more computational power as it perform intensive computational operations.

\section{Conclusion and future work}

In this paper, it is presented a study that correlates thermal comfort to environment induced stress through the use of ambient intelligence techniques and sensor networks. Results show promise on the identification of stressful situations as well as stress inference through the use of predictive algorithms. Though for predictive tasks and hypothesis making it is needed a learning phase for each person while stress identification can be made in real time without a learning context as it can be inferred by the context provided by other sensors. Future work should extend the capabilities of the purposed algorithms o take into consideration, user activities, emotion detection and emotion on stress assessment. From the literature, it said to be possible to distinguish emotion through temperature and pulse sensors.

\section{Acknowledgement}

This work was supported by the Spanish MEC and MICINN, as well as European Commission FEDER funds, under Grants TIN2009-14475-C04 and TIN201238341-C04-04. The work is also partially supported by a portuguese doctoral 
grant, SFRH/BD/78713/2011, issued by the Fundação da Ciência e Tecnologia (FCT) in Portugal.

\section{References}

1. M. Weiser, "The Computer for the Twenty-First Century," Scientific American, vol. 265, no. 3, pp. 94-104, 1991.

2. S. Ullah, H. Higgins, B. Braem, B. Latre, C. Blondia, I. Moerman, S. Saleem, Z. Rahman, and K. S. Kwak, "A comprehensive survey of Wireless Body Area Networks : on PHY, MAC, and Network layers solutions." Journal of medical systems, vol. 36, no. 3, pp. 1065-94, Jun. 2012.

3. M. Chen, S. Gonzalez, A. Vasilakos, H. Cao, and V. C. M. Leung, "Body Area Networks: A Survey," Mobile Networks and Applications, vol. 16, no. 2, pp. 171193, Aug. 2010.

4. C. O. N. of Excellence, "Recognizing Emotions using Wireless Sensor Networks," 2011.

5. W. H. Organization, "Stress at the workplace," 2013. [Online]. Available: http://www.who.int/occupational \_health/topics/stressatwp/en/

6. J.-P. Brun, "Work-related stress : scientific evidence-base of risk factors, prevention and costs."

7. J. Choi, B. Ahmed, and R. Gutierrez-Osuna, "Ambulatory Stress Monitoring with Minimally-Invasive Wearable Sensors," Comput. Sci. and Eng., Texas A\&M, 2010.

8. M. Tauseef, "Human Emotion Recognition Using Smart Sensors," Ph.D. dissertation, Massey University, 2012.

9. G. Acampora and V. Loia, "A proposal of ubiquitous fuzzy computing for Ambient Intelligence," Inf. Sci., vol. 178, no. 3, pp. 631-646, 2008.

10. W. Liu, Z. Lian, and Y. Liu, "Heart rate variability at different thermal comfort levels." European journal of applied physiology, vol. 103, no. 3, pp. 361-6, Jun. 2008.

11. A. D. Paola, S. Gaglio, G. L. Re, and M. Ortolani, "Sensor 9 k : A testbed for designing and experimenting with WSN-based ambient intelligence applications," Pervasive and Mobile Computing, vol. 8, no. 3, pp. 448-466, 2012.

12. $\mathrm{R}$ Core Team, R: A Language and Environment for Statistical Computing, $\mathrm{R}$ Foundation for Statistical Computing, Vienna, Austria, 2012. [Online]. Available: http://www.r-project.org/

13. M. Hall, H. National, E. Frank, G. Holmes, B. Pfahringer, P. Reutemann, and I. H. Witten, "The WEKA Data Mining Software : An Update," SIGKDD Explor. Newsl., vol. 11, no. 1, pp. 10-18, 2009.

14. A. Ortiz, F. Royo, R. Galindo, and T. Olivares, "I3ASensorBed: a testbed for wireless sensor networks," Tech. Rep., 2011.

15. PulseSensor, 2013. [Online]. Available: http://pulsesensor.myshopify.com/

16. P. O. Fanger, Thermal comfort: Analysis and applications in environmental engineering. Danish Technical Press, 1970.

17. P. Höppe, "The physiological equivalent temperature - a universal index for the biometeorological assessment of the thermal environment." International journal of biometeorology, vol. 43, no. 2, pp. 71-5, Oct. 1999.

18. M. Quazi and S. Mukhopadhyay, "Continuous monitoring of physiological parameters using smart sensors," 2011 Fifth International Conference on Sensing Technology, pp. 464-469, Nov. 2011. 